\title{
Conventional Versus Biological Therapy for Prevention of Postoperative Endoscopic Recurrence in Patients With Crohn's Disease: an International, Multicenter, and Observational Study
}

\author{
Paulo Gustavo Kotze ${ }^{1}$, Antonino Spinelli ${ }^{2}$, Rodolff Nunes da Silva ${ }^{1}$, Ivan Folchini de Barcelos ${ }^{1}$, Fábio Vieira \\ Teixeira $^{3}$, Rogério Saad-Hossne ${ }^{4}$, Idblan Carvalho de Albuquerque ${ }^{5}$, Marcia Olandoski ${ }^{1}$, Lorete Maria da \\ Silva Kotze ${ }^{1}$, Yasuo Suzuki ${ }^{6}$, Akihiro Yamada ${ }^{6}$, Ken Takeuchi $^{6}$, Matteo Sacchi $^{2}$, Takayuki Yamamoto ${ }^{7}$ \\ Colorectal Surgery Unit, Catholic University of Paraná, Curitiba, PR, Brazil', Colorectal and IBD Surgery Unit, Humanitas Research Hospital, \\ University of Milan, Milan, Italy², Clinica Gastrosaúde, Marília, SP, Brazil', Digestive Surgery Unit, Universidade Estadual Paulista Júlio de \\ Mesquita Filho (UNESP), Botucatu, SP, Brazil', Inflammatory Bowel Disease Outpatient Clinic, Heliópolis Hospital, São Paulo, SP, Brazil ${ }^{5}$, \\ Gastroenterology Unit, Sakura Hospital, Toho University, Chiba, Japan ${ }^{6}$, Inflammatory Bowel Disease Centre, Yokkaichi Hazu Medical Centre, \\ Yokkaichi, Japan
}

Background/Aims: Postoperative endoscopic recurrence (PER) occurs in nearly $80 \%$ of patients 1 year after ileocecal resection in patients with Crohn's disease (CD). Biological agents were more effective in reducing the rates of PER in comparison with conventional therapy, in prospective trials. The aim of this study was to compare the PER rates of biological versus conventional therapy after ileocecal resections in patients with CD in real-world practice. Methods: The MULTIPER (Multicenter International Postoperative Endoscopic Recurrence) database is a retrospective analysis of PER rates in CD patients after ileocecal resection, from 7 referral centers in 3 different countries. All consecutive patients who underwent ileocecal resections between 2008 and 2012 and in whom colonoscopies had been performed up to 12 months after surgery, were included. Recurrence was defined as Rutgeerts' score $\geq i 2$. The patients were allocated to either biological or conventional therapy after surgery, and PER rates were compared between the groups. Results: Initially, 231 patients were evaluated, and 63 were excluded. Of the 168 patients in the database, 96 received anti-tumor necrosis factor agents and 72 were treated with conventional therapy after resection. The groups were comparable regarding age, gender, and perianal disease. There was longer disease duration, more previous resections, and more open surgical procedures in patients on biologicals postoperatively. PER was identified in $25 / 96$ (26\%) patients on biological therapy and in 24/72 (33.3\%) patients on conventional therapy $(P=0.310)$. Conclusions: In this retrospective observational analysis from an international database, no difference was observed between biological and conventional therapy in preventing PER after ileocecal resections in CD patients. (Intest Res 2015;13:259-265)

Key Words: Crohn disease; Recurrence; Tumor necrosis factor-alpha

Received January 5, 2015. Revised February 18, 2015

Accepted March 2, 2015.

Correspondence to Paulo Gustavo Kotze, Rua Bruno Filgueira, 369 - cj.

1205, CEP 80240-220, Curitiba, PR, Brazil. Tel: +55-41-3243-0033, Fax:

+55-41-3243-0033, E-mail: pgkotze@hotmail.com

Financial support: None. Conflict of interest: None.

\section{INTRODUCTION}

Crohn's disease (CD) is a systemic, chronic inflammatory disorder of unknown etiology characterized by recurrences, and is incurable. Its evolution is asymmetrical, usually with focal involvement, and may affect the entire digestive tract. ${ }^{1}$ There is an ongoing discussion on the relevance of environ-

\footnotetext{
๑ Copyright 2015. Korean Association for the Study of Intestinal Diseases. All rights reserved.

This is an Open Access article distributed under the terms of the Creative Commons Attribution Non-Commercial License (http://creativecommons.org/licenses/by-nc/4.0)

which permits unrestricted non-commercial use, distribution, and reproduction in any medium, provided the original work is properly cited.
} 
mental, genetic, and immunological factors in the development of the disease. The gold standard for CD diagnosis is ileocolonoscopy with biopsies; complementary methodologies include capsule endoscopy, CT enterography, MRI, and ultrasound, as well as analysis of systemic and fecal biomarkers. $^{2-4}$

The main therapeutic options for the management of CD include: corticosteroids, antibiotics, 5-aminosalicylates (5-ASA), azathioprine (AZA), 6-mercaptopurine (6-MP), methotrexate, biological agents, and surgery. Despite advances in pharmacological therapy over the last decade, it is estimated that up to $75 \%$ of patients with CD in the terminal ileum and colon will still need surgery at some stage of the disease. ${ }^{5}$ Surgical procedures are the result of significant complications of CD, especially abscesses and perianal and abdominal fistulas, as well as symptomatic intestinal strictures, dysplasia, and cancer. ${ }^{6}$ Recurrence of CD remains among the most important challenges in its management, with $30-70 \%$ of patients reoperated within 10 years after the first resection. ${ }^{6}$ Nearly $70-90 \%$ of these patients will present with postoperative endoscopic recurrence (PER) between the first and third year after the surgical procedure. ${ }^{7,8}$

Many drugs have been tested as possible options for the prevention of recurrence after surgical resection in patients with CD, such as 5-ASA, antibiotics, corticosteroids, probiotics, thiopurines, and biological agents, such as infliximab (IFX) and adalimumab (ADA). ${ }^{9} 5$-ASA, antibiotics, and thiopurines are associated with limited efficacy, poor adherence to treatment, and possible adverse events. ${ }^{10}$ Biological agents have recently been compared to conventional therapy in prospective studies and in different case series. Major studies have found that this class of medications is superior in preventing PER compared to conventional therapy. ${ }^{11-15}$

Mostly owing to the lack of real-world data, the objective of this study was to compare PER rates among patients with CD treated with conventional therapy or biological agents (IFX and ADA) after ileocecal resections, in an international multicenter setting.

\section{METHODS}

\section{Study Design}

This multicenter, retrospective, and observational study was performed to determine PER rates in consecutive patients undergoing ileocecal resections, from January 2008 to January 2013; data were from seven referral centers for the management of $\mathrm{CD}$, located in three different countries (Brazil, Japan, and Italy). These data comprised the MULTIPER
(Multicenter International Postoperative Endoscopic Recurrence) database. This study was approved by the Research Ethics Committee of each institution involved.

\section{Inclusion and Exclusion Criteria}

The inclusion criteria for the study were: patients diagnosed with CD, age between 16 and 80 years, who had ileocecal resections (conventional or laparoscopic), and who underwent endoscopic evaluation (ileocolonoscopy) within at most 12 months after surgery. Exclusion criteria were patients with other IBDs (UC and indeterminate inflammatory disease), age under 16 and above 80 , who underwent ileocolonoscopy more than 12 months after surgery, as well as those who had incomplete or absent data in the medical charts.

\section{Patients' Characteristics}

Variables analyzed were age, gender, duration of CD, smoking, need for blood transfusion perioperatively, Montreal classification (at the time of diagnosis of CD), concomitant perianal disease, concomitant use of corticosteroids, AZA, or 6-MP, prior use of biologicals, previous surgical resections, residual CD, surgical approach (open or laparoscopic) and anastomotic technique (hand-sewn or stapled; end-to-end or side-to-side), early surgical complications, presence of granuloma, and extent of the surgical specimen.

\section{Definition of PER}

The occurrence of PER was determined according to the Rutgeerts' score, ${ }^{7}$ described in Table 1. PER was defined as a Rutgeerts' score $\geq i 2$, and patients with a score $\leq i 1$ were defined as having mucosal normality.

Table 1. Detailed Description of Rutgeerts' Score.

\begin{tabular}{cl}
\hline $\begin{array}{c}\text { Rutgeerts' } \\
\text { score }\end{array}$ & Endoscopic description of findings \\
\hline i0 & no lesions \\
i1 & $\leq 5$ aphthous ulcers \\
i2 & $>5$ aphthous ulcers with normal intervening mucosa, skip \\
& areas of larger lesions, or lesions confined to ileocolonic \\
& anastomosis \\
i3 & diffuse aphthous ileitis with diffusely inflamed mucosa \\
i4 & diffuse inflammation with larger ulcers, nodules and/or \\
& narrowing \\
\hline
\end{tabular}

Adapted From Rutgeerts et al. ${ }^{7}$ 


\section{Group Definition}

Following identification of patients who met the inclusion criteria from operative lists, the electronic medical records were reviewed, and a specific datasheet was completed. Patients were allocated into two groups, according to the treatment selected to prevent PER after the procedures: conventional or biological therapy. Patients receiving conventional therapy were treated with any type of non-biological medication (5-ASA, antibiotics, AZA, 6-MP). Patients in the biologicals group used anti-tumor necrosis factor agents (IFX or ADA), which could be administered concomitantly with conventional therapy.

The variables between the groups were compared. PER rates were determined at the first colonoscopies performed after surgery, and compared between these two groups. There was no centralized reading of the colonoscopies for classification of recurrence. The database included the classification by the Rutgeerts' score for every patient included in the electronic charts that were reviewed.

According to the practice at each of the 7 referral units, patients with high risk for recurrence (defined as having at least two of the following characteristics: previous resection, perforating abdominal disease, perianal CD, and smoking) were primarily treated with biologicals after surgery. Patients with low risk were primarily treated with conventional therapy.

\section{Statistical Analysis}

Statistical analysis was performed using SPSS software (IBM SPSS Statistics for Windows, Version 20.0. Armonk, NY: IBM Corp.). Fisher's exact test and Chi-Square test were used for assessing qualitative variables, and Student's $t$-test and non-parametric Mann-Whitney test for quantitative variables. Statistical significance was considered as $P<0.05$.

\section{RESULTS}

Initially, 231 patients were selected (85 from Brazil, 50 from Italy, and 96 from Japan). Of this initial sample, 63 were excluded owing to incomplete data in the medical records $(\mathrm{n}=18)$; first postoperative colonoscopy $>12$ months $(\mathrm{n}=39)$; or participation in other trials, in which the postoperative therapy was unknown $(n=6)$. Thus, 168 patients were included in the present study, 96 of whom received biological therapy, and 72 received conventional treatment after surgery. The study design is illustrated in Fig. 1.

The baseline characteristics of each group are shown in Table 2, for comparison. The data showed that patients managed with biological therapy after surgery had longer duration of CD; higher percentages of ileocolic involvement, according to the Montreal classification; higher rates of concomitant use of immunomodulators and previous resections; higher numbers of conventional procedures (open surgery, non-laparoscopic); and a higher incidence of granulomas, when compared to patients treated with conventional therapy. The time until the first postoperative colonoscopy was also recorded, and no difference was observed between the two groups.

With respect to the primary purpose of the study, PER was identified in 25/96 (26\%) patients on biological therapy, and in $24 / 72$ (33.3\%) on conventional therapy ( $P=0.310$; Fig. 2 ). The individual subscores from the Rutgeerts' score were also separately analyzed, and no statistical difference was found between the two groups ( $P=0.697$; Table 3$)$.

\section{DISCUSSION}

Surgical resection is still needed in many CD patients, regardless of recent advances in medical therapy (new drugs) or treatment strategies. During the natural history of the disease, approximately $70-80 \%$ of patients will require intes-

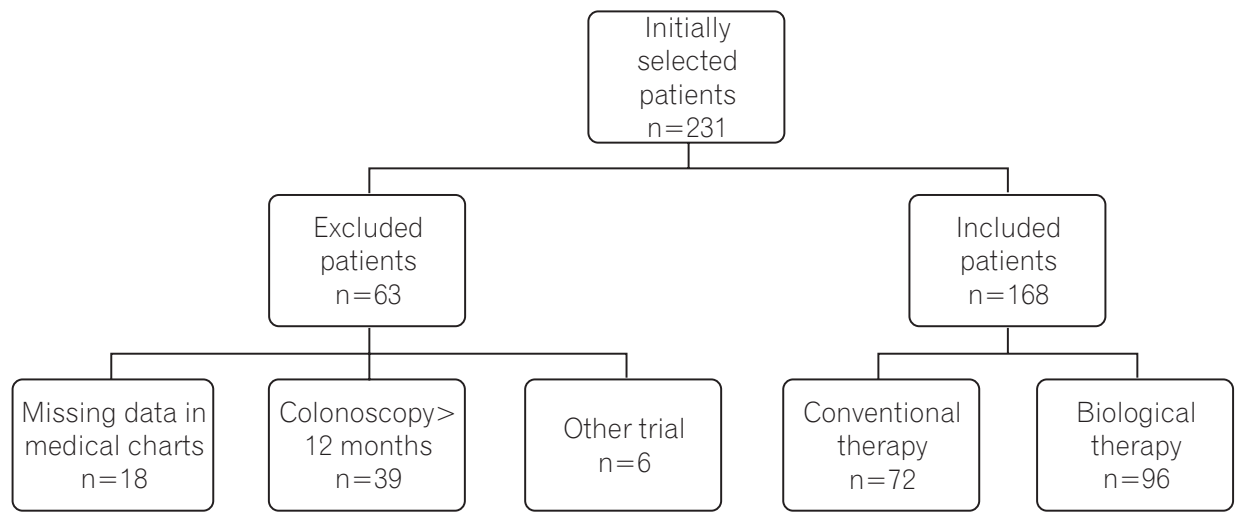

Fig. 1. Study design showing exclusion criteria and definition of samples in the analyzed groups. 
Table 2. Baseline Characteristics of the Patients, in the Two Groups Analyzed

\begin{tabular}{|c|c|c|c|}
\hline \multirow{2}{*}{ Variables } & \multicolumn{2}{|c|}{ Treatment (group) } & \multirow{2}{*}{$P$-value* } \\
\hline & Biological $(n=96)$ & Conventional $(n=72)$ & \\
\hline Age (yr) & $32.1 \pm 11.4$ & $34.3 \pm 12.6$ & 0.220 \\
\hline Duration of CD (mo) & $83(2-300)$ & $30(0-372)$ & $<0.001^{*}$ \\
\hline Surgical specimen (cm) & $34.7 \pm 21.4$ & $29.6 \pm 13.3$ & 0.059 \\
\hline Female gender & $37(38.5)$ & $32(44.4)$ & 0.526 \\
\hline \multicolumn{4}{|l|}{ Montreal classification } \\
\hline Age at diagnosis & & & 0.064 \\
\hline$<16$ yr (A1) & $10(10.4)$ & $5(6.9)$ & \\
\hline $17-40$ yr (A2) & $71(74)$ & $45(62.5)$ & \\
\hline$>40$ yr (A3) & $15(15.6)$ & $22(30.6)$ & \\
\hline Location & & & $0.015^{*}$ \\
\hline Ileal (L1) & $34(35.4)$ & $38(52.8)$ & \\
\hline Colonic (L2) & $6(6.3)$ & $0(0)$ & \\
\hline Ileocolonic (L3) & $56(58.3)$ & $34(47.2)$ & \\
\hline Behavior & & & 0.340 \\
\hline Inflammatory (B1) & $5(5.2)$ & $2(2.8)$ & \\
\hline Stricturing (B2) & $51(53.1)$ & $46(63.9)$ & \\
\hline Penetrating (B3) & $40(41.7)$ & $24(33.3)$ & \\
\hline Smoking & $13(13.5)$ & $12(16.7)$ & 0.663 \\
\hline Mean time of postoperative colonoscopy (mo) & $6.7 \pm 2.6$ & $6.6 \pm 2.7$ & 0.807 \\
\hline Perianal disease & $31(32.3)$ & $14(19.4)$ & 0.079 \\
\hline Concomitant steroids & $28(29.2)$ & $23(31.9)$ & 0.736 \\
\hline Concomitant AZA/6-MP & $37(38.5)$ & $16(22.2)$ & $0.029^{*}$ \\
\hline Preoperative biologicals & $50(52.1)$ & $3(4.2)$ & $<0.001^{*}$ \\
\hline Previous intestinal resections & $37(38.5)$ & $13(18.1)$ & $0.006^{*}$ \\
\hline Blood transfusion & $9(10.6)$ & $7(17.5)$ & 0.389 \\
\hline Residual CD after surgery & $16(16.7)$ & $11(15.3)$ & 0.836 \\
\hline Conventional procedure & $72(75)$ & $34(47.2)$ & $<0.001^{*}$ \\
\hline Hand-sewn anastomosis & $23(24)$ & $20(27.8)$ & 0.596 \\
\hline Side-to-side anastomosis & $73(76)$ & $56(77.8)$ & 0.855 \\
\hline Early surgical complications & $15(15.6)$ & $13(18.1)$ & 0.682 \\
\hline Abdominal Sepsis & $9(9.4)$ & $6(8.3)$ & 1.000 \\
\hline Anastomotic dehiscence & $8(8.3)$ & $4(5.6)$ & 0.559 \\
\hline Granuloma & $52(54.2)$ & $24(33.3)$ & $0.008^{*}$ \\
\hline
\end{tabular}

Values are presented as a $n(\%)$ or mean \pm SD.

*Indicates a statistical difference between the groups in relation to the variable analyzed.

AZA, azathioprine; 6-MP, 6-mercaptopurine.

tinal resection within 10 years of diagnosis., ${ }^{8,16}$ PER is a common event after surgery, occurring in up to $80 \%$ of patients after 1 year. ${ }^{9}$

Thus, the use of colonoscopy and subsequent application of the Rutgeerts' score up to 12 months after surgery, helps to identify patients with early PER, and thus optimize drug therapy to achieve better control of disease activity. ${ }^{8}$

Several drugs have been studied for the prevention of PER: 


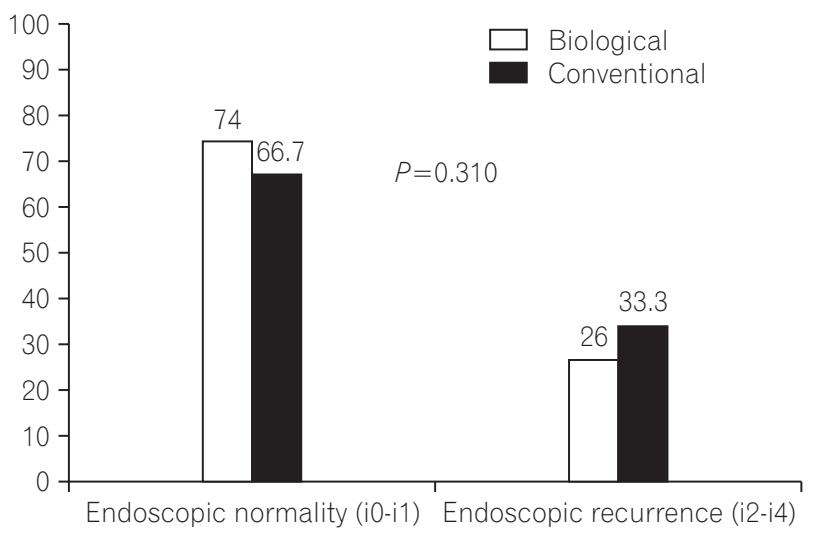

Fig. 2. The main results of the study. Absence of statistical difference between groups considering postoperative endoscopic recurrence rates $(P=0.310)$

Table 3. Individual Subscores of the Rutgeerts' Score Comparing the Two Groups

\begin{tabular}{ccc}
\hline Rutgeerts' score & Biological & Conventional \\
\hline i-0 & $32(33.3)$ & $24(33.3)$ \\
i-1 & $39(40.6)$ & $24(33.3)$ \\
i-2 & $12(12.5)$ & $14(19.4)$ \\
i-3 & $9(9.4)$ & $8(11.1)$ \\
i-4 & $4(4.2)$ & $2(2.8)$ \\
Total & $96(100)$ & $72(100)$ \\
\hline
\end{tabular}

Values are presented as $n(\%)$.

metronidazole, probiotics, AZA, and more recently, biological agents (IFX and ADA). Each medication has its indication based on clinical criteria: patients with a lower risk of recurrence can be managed with conventional therapy, and patients with a higher risk require more powerful drugs, such as biological agents, for better long-term results. ${ }^{8}$

Strategies for postoperative management to prevent PER may help maintain longer endoscopic remission. Conventional therapy has proved to be effective and should be used in patients with a lower risk of endoscopic recurrence. ${ }^{17,18}$

Previous prospective studies with biological agents have suggested greater efficacy for the prevention of PER in relation to conventional treatment in patients at high risk for recurrence (mainly smokers, and patients with penetrating disease and previous resections). Most of these studies were performed in small patient samples with a short follow-up period. Few observational studies have been published. ${ }^{19-23}$

The present study, a real-world observational case series with patients from 3 different countries, showed no difference in PER rates between patients treated with biological or conventional therapy ( $26 \%$ vs. $33.3 \%, P=0.310$ ). A likely reason for these results is the lack of complete homogeneity between patients in the study groups. Individuals who used IFX or ADA after surgery had a longer duration of CD and higher rate of concomitant immunomodulator use, in addition to a higher percentage of conventional operations (possibly due to more severe disease); these probably characterized a group of patients with worse prognosis over time, and consequently a higher chance of developing PER. Furthermore, patients from the biological group had a higher rate of prior resections for $\mathrm{CD}$, and a higher frequency of granulomas, factors reported in the literature that similarly suggest a higher risk for recurrence. ${ }^{8,9}$ These factors may have contributed to a significant increase in PER rates in this group of patients.

The present study suggests that the lack of statistical difference was due to a higher PER rate in the group of patients receiving biological treatment, when prospective studies suggest the opposite. ${ }^{21}$ Regueiro et al., in a prospective randomized study comparing conventional treatment with IFX, identified PER after 1 year in only $9.1 \%$ of patients who used biological therapy. ${ }^{12}$ Savarino et al. compared the use of ADA with AZA and 5-ASA, and the rate of PER 2 years after ileocolic resections in the group using ADA was only $6.3 \%{ }^{22}$ In the present study, the observed 26\% PER rate may also be explained by the fact that not all patients had a "curative" resection, without residual CD; among other factors, this is a totally different population from that of the previously mentioned studies.

A recently published randomized trial compared two different strategies in postoperative follow-up of patients submitted to ileocecal resections. In this study (the PostOperative Crohn's Endoscopic Recurrence [POCER] Trial), tailoring therapy based on the findings of colonoscopy performed 6 months postoperatively led to lower PER rates; this was in comparison to the best prescribed postoperative regimen, without interventional endoscopic examination, in a longer follow-up of 18 months. ${ }^{24}$ Our observational study demonstrated that the mean time for the first postoperative colonoscopy was approximately 6 months in both groups, in accordance with the conclusions and the recommendations of the ideal strategy for these patients, as described in this important trial.

The limitations of a multicenter, international, and observational retrospective study also contributed to the absence of differences and failure to confirm the primary hypothesis of this study. Patients were not followed in the same unit, and centralized reading of postoperative endoscopic examina- 
tions was not conducted; this may have become a source of bias in the interpretation and application of Rutgeerts' score, in defining the presence or absence of PER. On the other hand, the exclusion of patients who underwent colonoscopy more than 12 months after surgery may have balanced the results.

Another possible limitation of the present study was its observational design. The decision on which drug was used in the prevention of PER was not standardized; data in the medical records of these patients were compiled according to the findings in real-world clinical practice. The population was heterogeneous, mixing patients with low and high risk for recurrence. Patients with low risk were primarily managed with conventional therapy, while those with high risk for PER primarily used biologicals. There was also a combination of patients who used biologicals only after surgical procedures, along with patients who had already been on IFX or ADA before surgery, which may have biased data analysis.

The groups were not completely comparable for some important variables, with the biologicals group at a significantly higher risk for development of PER. Moreover, the absence of a significant difference in this international case series, with heterogeneous groups, may raise the question whether the postoperative use of biologicals could not counterbalance the intrinsically worse prognostic factors of this population. Early detection of PER and prompt prospective dose optimization in this group of patients could reduce the rates of PER in patients on ADA or IFX, and consequently highlight the differences between conventional and biological therapy in this scenario.

The greatest contribution of real-world observation, as represented by this study, is that postoperative management of CD can be further improved. Moreover, a better selection of patients for a determined therapy, based on risk factors, might counterbalance the differences between these two groups of patients.

In summary, in this multicenter observational study, PER prevention after ileocolectomies in CD patients was not different between patients receiving biological and conventional therapy. Prospective studies with better patient selection still indicate biological therapy best prevents PER in patients at high risk for recurrence. However, our data show that in clinical practice, the postoperative management of CD can be improved further.

\section{ACKNOWLEDGEMENT}

We would like to thank the library personnel from the Cajuru University hospital (PUCPR) for their assistance in accessing the articles referenced in this paper.

\section{REFERENCES}

1. Baumgart DC, Sandborn WJ. Crohn's disease. Lancet 2012;380:1590-1605.

2. Dionisio PM, Gurudu SR, Leighton JA, et al. Capsule endoscopy has a significantly higher diagnostic yield in patients with suspected and established small-bowel Crohn's disease: a metaanalysis. Am J Gastroenterol 2010;105:1240-1248.

3. Panés J, Bouzas R, Chaparro M, et al. Systematic review: the use of ultrasonography, computed tomography and magnetic resonance imaging for the diagnosis, assessment of activity and abdominal complications of Crohn's disease. Aliment Pharmacol Ther 2011;34:125-145.

4. Lewis JD. The utility of biomarkers in the diagnosis and therapy of inflammatory bowel disease. Gastroenterology 2011;140:1817-1826.

5. Swoger JM, Regueiro M. Preventive therapy in postoperative Crohn's disease. Curr Opin Gastroenterol 2010;26:337-343.

6. Larson DW, Pemberton JH. Current concepts and controversies in surgery for IBD. Gastroenterology 2004;126:1611-1619.

7. Rutgeerts P, Geboes K, Vantrappen G, Beyls J, Kerremans R, Hiele M. Predictability of the postoperative course of Crohn's disease. Gastroenterology 1990;99:956-963.

8. De Cruz P, Kamm MA, Prideaux L, Allen PB, Desmond PV. Postoperative recurrent luminal Crohn's disease: a systematic review. Inflamm Bowel Dis 2012;18:758-777.

9. Buisson A, Chevaux JB, Bommelaer G, Peyrin-Biroulet L. Diagnosis, prevention and treatment of postoperative Crohn's disease recurrence. Dig Liver Dis 2012;44:453-460.

10. Doherty G, Bennett G, Patil S, Cheifetz A, Moss AC. Interventions for prevention of post-operative recurrence of Crohn's disease. Cochrane Database Syst Rev doi: 10.1002/14651858. CD006873.pub2. Published online 7 October 2009.

11. Yamamoto T, Umegae S, Matsumoto K. Impact of infliximab therapy after early endoscopic recurrence following ileocolonic resection of Crohn's disease: a prospective pilot study. Inflamm Bowel Dis 2009;15:1460-1466.

12. Regueiro M, Schraut W, Baidoo L, et al. Infliximab prevents Crohn's disease recurrence after ileal resection. Gastroenterology 2009;136:441-450. 
13. Papamichael K, Archavlis E, Lariou C, Mantzaris GJ. Adalimumab for the prevention and/or treatment of post-operative recurrence of Crohn's disease: a prospective, two-year, single center, pilot study. J Crohns Colitis 2012;6:924-931.

14. Sorrentino D, Paviotti A, Terrosu G, Avellini C, Geraci M, Zarifi D. Low-dose maintenance therapy with infliximab prevents postsurgical recurrence of Crohn's disease. Clin Gastroenterol Hepatol 2010;8:591-599.

15. Savarino E, Dulbecco P, Bodini G, Assandri L, Savarino V. Prevention of postoperative recurrence of Crohn's disease by Adalimumab: a case series. Eur J Gastroenterol Hepatol 2012;24:468-470.

16. Rutgeerts P, Geboes K, Vantrappen G, Kerremans R, Coenegrachts JL, Coremans G. Natural history of recurrent Crohn's disease at the ileocolonic anastomosis after curative surgery. Gut 1984;25:665-672.

17. Peyrin-Biroulet L, Deltenre P, Ardizzone S, et al. Azathioprine and 6-mercaptopurine for the prevention of postoperative recurrence in Crohn's disease: a meta-analysis. Am J Gastroenterol 2009;104:2089-2096.

18. Van Assche G, Dignass A, Reinisch W, et al. The second European evidence-based Consensus on the diagnosis and management of Crohn's disease: Special situations. J Crohns Colitis 2010;4:63-101.
19. Yoshida K, Fukunaga K, Ikeuchi H, et al. Scheduled infliximab monotherapy to prevent recurrence of Crohn's disease following ileocolic or ileal resection: a 3-year prospective randomized open trial. Inflamm Bowel Dis 2012;18:1617-1623.

20. Sorrentino D, Terrosu G, Paviotti A, et al. Early diagnosis and treatment of postoperative endoscopic recurrence of Crohn's disease: partial benefit by infliximab—a pilot study. Dig Dis Sci 2012;57:1341-1348.

21. Kotze PG, Saad-Hossne R. Biological therapy for the prevention and treatment of postoperative endoscopic recurrence in Crohn's disease: time for acceptance? Intest Res 2013;11:256260.

22. Savarino E, Bodini G, Dulbecco P, et al. Adalimumab is more effective than azathioprine and mesalamine at preventing postoperative recurrence of Crohn's disease: a randomized controlled trial. Am J Gastroenterol 2013;108:1731-1742.

23. Yamamoto T. Prevention of recurrence after surgery for Crohn's disease: efficacy of infliximab. World J Gastroenterol 2010;16:5405-5410.

24. De Cruz P, Kamm MA, Hamilton AL, et al. Crohn's disease management after intestinal resection: a randomised trial. Lancet 2015;385:1406-1417. 